ANNALES

POLONICI MATHEMATICI

$89.2(2006)$

\title{
Normality and value sharing with a linear differential polynomial
}

by IndRAJit LAhiri (Kalyani) and Shyamali Dewan (Kolkata)

\begin{abstract}
We prove some normality criteria for a family of meromorphic functions and as an application we prove a value distribution theorem for a differential polynomial.
\end{abstract}

1. Introduction, definitions and results. Let $\mathbb{C}$ be the open complex plane and $\mathfrak{D} \subset \mathbb{C}$ be a domain. A family $\mathfrak{F}$ of meromorphic functions defined in $\mathfrak{D}$ is said to be normal, in the sense of Montel, if for every sequence $\left\{f_{n}\right\} \subset \mathfrak{F}$ there exists a subsequence $\left\{f_{n_{j}}\right\}$ such that $\left\{f_{n_{j}}\right\}$ converges spherically and uniformly on compact subsets of $\mathfrak{D}$ to a meromorphic function or $\infty$.

$\mathfrak{F}$ is said to be normal at a point $z_{0} \in \mathfrak{D}$ if there exists a neighbourhood of $z_{0}$ in which $\mathfrak{F}$ is normal. It is well known that $\mathfrak{F}$ is normal in $\mathfrak{D}$ if and only if it is normal at every point of $\mathfrak{D}$.

Let $f$ and $g$ be two meromorphic functions defined in $\mathfrak{D}$. For $a \in \mathbb{C} \cup\{\infty\}$ we say that $f$ and $g$ share the value a IM (ignoring multiplicity) if the $a$ points of $f$ and $g$ coincide in locations only, not necessarily in multiplicities.

For a meromorphic function $f$ we denote by $f^{\#}$ the spherical derivative of $f$, given by

$$
f^{\#}(z)=\frac{\left|f^{\prime}(z)\right|}{1+|f(z)|^{2}} .
$$

Also, by $\Delta$ we denote the unit disc $|z|<1$.

In 1992 W. Schwick [15] first established a connection between the normality and value sharing. He proved the following theorem.

THEOREM A ([15]). Let $\mathfrak{F}$ be a family of meromorphic functions in a domain $\mathfrak{D} \subset \mathbb{C}$ and $a_{1}, a_{2}, a_{3}$ be distinct complex numbers. If for every $f \in \mathfrak{F}$, $f$ and $f^{\prime}$ share $a_{1}, a_{2}, a_{3}$ IM in $\mathfrak{D}$ then $\mathfrak{F}$ is normal in $\mathfrak{D}$.

2000 Mathematics Subject Classification: 30D45, 30D35.

Key words and phrases: meromorphic function, differential polynomial, normality. 
After the work of Schwick [15] it has become a popular problem to investigate the relation between normality and sharing values.

In 1999 Y. Xu [16] proved the following result.

THEOREM B ([16]). Let $\mathfrak{F}$ be a family of holomorphic functions in a domain $\mathfrak{D} \subset \mathbb{C}$ and $b$ be a nonzero complex number. If $f$ and $f^{\prime}$ share $0, b$ IM in $\mathfrak{D}$ for every $f \in \mathfrak{F}$ then $\mathfrak{F}$ is normal in $\mathfrak{D}$.

In 2000 X. Pang and L. Zalcman [12] proved the following result, which improves Theorems A and B.

THEOREM C ([12]). Let $\mathfrak{F}$ be a family of meromorphic functions in a domain $\mathfrak{D} \subset \mathbb{C}$ and $a_{1}, a_{2}$ be distinct complex numbers. If for every $f \in \mathfrak{F}$, $f$ and $f^{\prime}$ share $a_{1}, a_{2}$ IM in $\mathfrak{D}$ then $\mathfrak{F}$ is normal in $\mathfrak{D}$.

At this stage two natural questions may be asked:

1. What would be if $f$ and $f^{\prime}$ share a single value?

2. What would be if $f^{\prime}$ is replaced by $f^{(k)}$ ?

For Question 1 the following result of W. C. Lin and H. X. Yi [11] may be noted.

THEOREM D ([11]). Let $\mathfrak{F}$ be a family of meromorphic functions in $\Delta$. If there exist complex numbers $a$ and $b(b \neq 0$ and $a / b$ not a positive integer $)$ such that for every $f \in \mathfrak{F}, f$ and $f^{\prime}$ share a IM in $\Delta$ and $|f(z)-a| \geq \varepsilon$ whenever $f^{\prime}(z)=b$, where $\varepsilon$ is a positive number, then $\mathfrak{F}$ is normal in $\Delta$.

For Question 2, H. Chen and M. Fang [3] proved the following result.

THEOREM E ([3]). Let $\mathfrak{F}$ be a family of meromorphic functions in a domain $\mathfrak{D} \subset \mathbb{C}, k \geq 2$ be an integer and $a, b, c$ be complex numbers such that $b \neq a$. If for each $f \in \mathfrak{F}, f$ and $f^{(k)}$ share $a, b$ IM in $\mathfrak{D}$ and zeros of $f-c$ have multiplicity at least $1+k$ then $\mathfrak{F}$ is normal in $\mathfrak{D}$.

The following result of M. Fang and L. Zalcman [5] improved Theorem E.

THEOREM $\mathrm{F}([5])$. Let $\mathfrak{F}$ be a family of meromorphic functions in a domain $\mathfrak{D} \subset \mathbb{C}, k \geq 2$ be an integer and $a, b, c$ be complex numbers such that $b \neq a$. If for each $f \in \mathfrak{F}, f$ and $f^{(k)}$ share $a, b$ IM in $\mathfrak{D}$ and zeros of $f-c$ have multiplicity at least $k$ then $\mathfrak{F}$ is normal in $\mathfrak{D}$.

Theorem $\mathrm{F}$ is a consequence of the following theorem, also due to Fang and Zalcman [5].

THEOREM G ([5]). Let $\mathfrak{F}$ be a family of meromorphic functions in a domain $\mathfrak{D} \subset \mathbb{C}, k$ be a positive integer and $a, b, c, d$ be complex numbers such that $b \neq a, 0$ and $c \neq 0$. If , for each $f \in \mathfrak{F}$, all zeros of $f-d$ have multiplicity at least $k, f$ and $f^{(k)}-a$ share 0 IM and $f(z)=c$ whenever $f^{(k)}(z)=b$, then $\mathfrak{F}$ is normal in $\mathfrak{D}$ for $k \geq 2$, and for $k=1$ so long as $a \neq(1+m) b$, $m=1,2, \ldots$. 
In this paper we investigate the situation when the derivative is replaced by a linear differential polynomial with constant coefficients generated by $f$. Throughout the paper we denote by $H_{k}(f)=H_{k}\left(f ; a_{1}, \ldots, a_{k}\right)$ a linear differential polynomial generated by a meromorphic function $f$ of the following form:

$$
H_{k}(f)=H_{k}\left(f ; a_{1}, \ldots, a_{k}\right)=a_{k} f^{(k)}+a_{k-1} f^{(k-1)}+\cdots+a_{1} f^{(1)}
$$

where $k$ is a positive integer and $a_{1}, \ldots, a_{k} \neq 0$ are constants.

We now state the main result of the paper.

THEOREM 1.1. Let $\mathfrak{F}$ be a family of meromorphic functions in a domain $\mathfrak{D} \subset \mathbb{C}$ and $a, b, c, d$ be finite complex numbers such that $c \neq 0$. If there exists a differential polynomial $H_{k}(f)=H_{k}\left(f ; a_{1}, \ldots, a_{k}\right)$ such that for each $f \in \mathfrak{F}$,

(i) $f-d$ does not have any zero with multiplicity less than $k$,

(ii) $f-a$ and $H_{k}(f)-b$ share the value $0 I M$,

(iii) $|f(z)-a| \geq \varepsilon$ whenever $H_{k}(f)=c$, where $\varepsilon$ is a positive number, then $\mathfrak{F}$ is normal in $\mathfrak{D}$ for $k \geq 2$, and for $k=1$ so long as $b / c \neq 1+m$ for any positive integer $m$.

The following example shows that condition (i) of Theorem 1.1 is essential.

EXAmple 1.1. Let $f_{n}(z)=n e^{z}-n e^{-z}+1$ for $n=1,2, \ldots$ and $\mathfrak{D}=\mathbb{C}$. We choose $k=2, a=1, b=0, c=1$ and $\varepsilon=1$. Then for any given finite complex number $d$,

$$
f_{n}(z)-d=\frac{n e^{2 z}+(1-d) e^{z}-n}{e^{z}}
$$

has only simple zeros in $\mathfrak{D}$ (except possibly for only one value of $n$ for which $d=1 \pm 2 n i)$. Also $f_{n}(z)-a$ and $f_{n}^{(2)}(z)-b$ share 0 IM and $\left|f_{n}(z)-a\right|=2>\varepsilon$ whenever $f_{n}^{(2)}(z)=c$. Since $f_{n}^{\#}(0)=n \rightarrow \infty$ as $n \rightarrow \infty$, by Marty's criterion the family $\left\{f_{n}\right\}$ is not normal in $\mathfrak{D}$.

The following example shows that condition (ii) of Theorem 1.1 is essential.

EXAmple 1.2. Let $f_{n}(z)=n z^{2}$ for $n=1,2, \ldots$ and $\mathfrak{D}=\Delta$. We choose $k=2, a=0, b=0, d=0$ and $c=1$. Then $f_{n}(z)-d$ has no zero of multiplicity less than $k, f_{n}^{(2)}(z)=2 n$ does not assume the value $c$, so that condition (iii) of Theorem 1.1 is satisfied but $f_{n}(z)$ and $f_{n}^{(2)}(z)$ do not share the value $a=b=0$. Since $f_{n}(0)=0$ for $n=1,2, \ldots$ and for $z \neq 0$, $f_{n}(z) \rightarrow \infty$ as $n \rightarrow \infty$, it follows that the family $\left\{f_{n}\right\}$ is not normal in $\mathfrak{D}$.

The following example shows that condition (iii) of Theorem 1.1 is essential. 
EXAmPLe 1.3. Let $f(z)=e^{n z}$ for $n=1,2, \ldots$ and $\mathfrak{D}=\Delta$. We choose $k=2, a=0, b=0, c=1$ and $d=0$. Then conditions (i) and (ii) of Theorem 1.1 are satisfied. Also we see that $f_{n}^{(2)}(z)=c$ implies $\left|f_{n}(z)-a\right|=$ $1 / n^{2} \rightarrow 0$ as $n \rightarrow \infty$ so that we cannot find any $\varepsilon>0$ for which condition (iii) is satisfied. Since $f_{n}^{\#}(0)=n / 2 \rightarrow \infty$ as $n \rightarrow \infty$, by Marty's criterion the family $\left\{f_{n}\right\}$ is not normal in $\mathfrak{D}$.

The following example shows that the condition $c \neq 0$ cannot be removed from Theorem 1.1.

EXAMPLE 1.4. Let $f_{n}(z)=e^{n z}-a / n+a$ for $n=1,2, \ldots$ and $\mathfrak{D}=\Delta$. Then $f_{n}$ and $f_{n}^{(1)}$ share the value $a$ IM. Also $f_{n}^{(1)}(z) \neq 0$ in $\mathfrak{D}$ so that condition (iii) of Theorem 1.1 is satisfied for $c=0$. Since

$$
f_{n}^{\#}(0)=\frac{n}{1+|a / n+a|} \rightarrow \infty \quad \text { as } n \rightarrow \infty,
$$

by Marty's criterion the family $\left\{f_{n}\right\}$ is not normal in $\mathfrak{D}$.

The following example shows that for $k=1$ the condition " $b / c \neq 1+m$ for any positive integer $m$ " of Theorem 1.1 is essential.

EXAMPLE 1.5. Let $b$ and $c$ be two nonzero numbers such that $b=$ $(1+m) c$, where $m$ is a positive integer. Also let $\left\{\alpha_{n}\right\}$ be a sequence of numbers converging to 0 and $\left|\alpha_{n}\right|<1$ for $n=1,2, \ldots$. We suppose that $\mathfrak{D}=\Delta$ and, for $n=1,2, \ldots$,

$$
f_{n}(z)=c\left(z-\alpha_{n}\right)+\frac{A\left(\alpha_{n}\right)^{m}}{m\left(z-\alpha_{n}\right)^{m}},
$$

where $A$ is a nonzero constant. Then

$$
f_{n}^{(1)}(z)=c-\frac{A\left(\alpha_{n}\right)^{m}}{\left(z-\alpha_{n}\right)^{m+1}}
$$

so that $f_{n}^{(1)}(z)$ does not assume the value $c$ and so condition (iii) of Theorem 1.1 is satisfied. Also

$$
\begin{aligned}
f_{n}(z) & =\frac{m c\left(z-\alpha_{n}\right)^{m+1}+A\left(\alpha_{n}\right)^{m}}{m\left(z-\alpha_{n}\right)^{m}}, \\
f_{n}^{(1)}(z)-b & =-\frac{m c\left(z-\alpha_{n}\right)^{m+1}+A\left(\alpha_{n}\right)^{m}}{m\left(z-\alpha_{n}\right)^{m+1}}
\end{aligned}
$$

so that $f_{n}$ and $f_{n}^{(1)}$ share 0 IM. Again

$$
\begin{aligned}
f_{n}^{\#}(0) & =\frac{\left|c+(-1)^{m+2} / \alpha_{n}\right|}{1+\left|-c \alpha_{n}+(-1)^{m} A / m\right|^{2}} \\
& \geq \frac{1 /\left|\alpha_{n}\right|-|c|}{1+\left\{|c|\left|\alpha_{n}\right|+|A| / m\right\}^{2}} \rightarrow \infty \quad \text { as } n \rightarrow \infty .
\end{aligned}
$$

Hence by Marty's criterion the family $\left\{f_{n}\right\}$ is not normal in $\mathfrak{D}$. 
The following corollary not only extends Theorem $\mathrm{G}$ to a linear differential polynomial but also removes the hypothesis $a \neq b$.

COROLlaRY 1.1. Let $\mathfrak{F}$ be a family of meromorphic functions in a domain $\mathfrak{D} \subset \mathbb{C}$ and $a, b, c, d, \alpha$ be finite complex numbers such that $b \neq 0$ and $c \neq \alpha$. If there exists a differential polynomial $H_{k}(f)=H_{k}\left(f ; a_{1}, \ldots, a_{k}\right)$ such that for each $f \in \mathfrak{F}$,

(i) $f-d$ does not have any zero of multiplicity less than $k$,

(ii) $f-\alpha$ and $H_{k}(f)-a$ share the value $0 I M$,

(iii) $f(z)=c$ whenever $H_{k}(f)=b$,

then $\mathfrak{F}$ is normal in $\mathfrak{D}$ for $k \geq 2$, and for $k=1$ so long as $a / b \neq 1+m$ for any positive integer $m$.

REMARK 1.1. If we choose $a=b$ then from conditions (ii) and (iii) of Corollary 1.1 it is obvious that $\alpha$ and $a$ are lacunary values of $f \in \mathfrak{F}$ and $H_{k}(f)$ respectively.

The following example shows that in Corollary 1.1 the condition $b \neq 0$ is essential.

EXAMPLE 1.6. Let $f_{n}(z)=e^{n z}$ for $n=1,2, \ldots$ and $\mathfrak{D}=\Delta$. We choose $\alpha=a=b=d=0$. Then $f_{n}(z)-d$ does not have any zero and for any positive integer $k, f_{n}(z)$ and $f_{n}^{(k)}(z)-a$ share the value 0 IM. Since $f_{n}^{(k)}(z) \neq b$, it follows that condition (iii) of Corollary 1.1 is satisfied for any complex number $c$. Since $f_{n}^{\#}(0)=n / 2 \rightarrow \infty$ as $n \rightarrow \infty$, by Marty's criterion the family $\left\{f_{n}\right\}$ is not normal in $\mathfrak{D}$.

The following corollary improves Theorems $\mathrm{C}$ and F.

CoROLlaRY 1.2. Let $\mathfrak{F}$ be a family of meromorphic functions in a domain $\mathfrak{D} \subset \mathbb{C}$ and $a, b, c$ be finite numbers such that $a \neq b$. If there exists $a$ differential polynomial $H_{k}(f)=H_{k}\left(f ; a_{1}, \ldots, a_{k}\right)$ such that for each $f \in \mathfrak{F}$,

(i) $f-c$ does not have any zero of multiplicity less than $k$,

(ii) $f$ and $H_{k}(f)$ share the values $a$ and $b I M$,

then $\mathfrak{F}$ is normal in $\mathfrak{D}$.

For standard definitions and notations we refer to [7] and [14].

2. Lemmas. In this section we present some necessary lemmas.

LEMMA 2.1 ([13]). Let $\mathfrak{F}$ be a family of meromorphic functions in $\Delta$ having no zero of multiplicity less than $k$. Suppose there exists a number $A \geq 1$ such that $\left|f^{(k)}(z)\right| \leq A$ whenever $f(z)=0$. If $\mathfrak{F}$ is not normal in $\Delta$ then there exist, for each $\alpha(0 \leq \alpha \leq k)$,

(i) a number $r, 0<r<1$, 
(ii) points $z_{n},\left|z_{n}\right|<r$,

(iii) functions $f_{n} \in \mathfrak{F}$ and

(iv) positive numbers $\varrho_{n}, \varrho_{n} \rightarrow 0$,

such that $g_{n}(\xi)=\varrho_{n}^{-\alpha} f_{n}\left(z_{n}+\varrho_{n} \xi\right) \rightarrow g(\xi)$ spherically and locally uniformly to a nonconstant meromorphic function $g$ in $\mathbb{C}$, all of whose zeros have multiplicity at least $k$ and $g^{\#}(\xi) \leq g^{\#}(0)=k A+1$. Moreover the order of $g$ is at most 2 .

LEMMA $2.2([5])$. Let $f$ be a meromorphic function of finite order and $a, b \neq 0$ be distinct complex numbers and $k \geq 2$ be an integer. If $f$ has no zero of multiplicity less than $k, f$ and $f^{(k)}-a$ share the value 0 IM and $f^{(k)}$ does not assume the value $b$, then $f$ is a constant.

LEMMA $2.3([5,8,11])$. Let $f$ be a nonconstant meromorphic function of finite order and let $a, b \neq 0$ be distinct complex numbers. If $f$ and $f^{(1)}-a$ share the value 0 IM and $f^{(1)}$ does not assume the value $b$ in $\mathbb{C}$ then

$$
f(z)=b(z-d)+\frac{A}{m(z-d)^{m}} \quad \text { and } \quad a=(1+m) b
$$

for some $d \in \mathbb{C}$ and some positive integer $m$.

LEMMA 2.4 ([9]). Let $f$ be a nonconstant rational function, and $k$ and $\lambda \geq 2$ be positive integers such that

(i) $f$ has no zero of multiplicity less than $\lambda$ and the number of zeros of $f$ (counted with multiplicities), if there are any, is not less than $1+k$,

(ii) if $f$ has any pole then the number of poles of $f$ (counted with multiplicities $)$ is greater than $k /(\lambda-1)$.

Then for every complex number $a \neq 0, \infty$, the function $f^{(k)}+a$ has at least one zero.

LEMMA 2.5. Let $f$ be a nonconstant rational function having no zero and $k$ be a positive integer. Then for every complex number $a \neq 0, \infty$, the function $f^{(k)}+$ a has at least one zero.

Proof. Since $f$ has no zero, choosing $\lambda=k+2$ in Lemma 2.4 we obtain the result.

LEMMA 2.6 ([7, p. 60]). Suppose that $f$ is meromorphic and transcendental in $\mathbb{C}$. Then for any positive integer $k$,

$$
T(r, f) \leq(2+1 / k) N(r, 0 ; f)+(2+2 / k) \bar{N}\left(r, a ; f^{(k)}\right)+S(r, f),
$$

where $a \neq 0, \infty$ is a complex number.

LEMMA 2.7 ([2]). Let $f$ be a meromorphic function of finite order. If $f$ has only finitely many critical values then it has only finitely many asymptotic values. 
Lemma 2.8 ([1]). Let $f$ be a transcendental meromorphic function such that $f(0) \neq \infty$ and let the set of finite critical and asymptotic values of $f$ be bounded. Then there exists $R>0$ such that

$$
\left|f^{\prime}(z)\right| \geq \frac{|f(z)|}{2 \pi|z|} \log \frac{|f(z)|}{R}
$$

for all $z \in \mathbb{C} \backslash\{0\}$ which are not poles of $f$.

Lemma $2.9([6,10])$. Let $f$ be a nonconstant meromorphic function in $\mathbb{C}$ and $k \geq 2$ be an integer. If $f$ and $f^{(k)}$ do not assume the value 0 in $\mathbb{C}$ then either $f(z)=e^{A z+B}$ or $f(z)=(A z+B)^{-m}$, where $A \neq 0$ and $B$ are constants and $m$ is a positive integer.

Lemma $2.10([4])$. Let $f$ be a meromorphic function in $\mathbb{C}$. If there exists a constant $M>0$ such that $f^{\#}(z) \leq M$ in $\mathbb{C}$ then the order of $f$ is at most 2 .

Lemma 2.11 ([7, p. 57]). Let $f$ be a nonconstant meromorphic function in $\mathbb{C}$ and $H_{k}(f)$ be nonconstant. Then for any complex number $a \neq 0, \infty$,

$$
T(r, f) \leq \bar{N}(r, \infty ; f)+N(r, 0 ; f)+\bar{N}\left(r, a ; H_{k}(f)\right)+S(r, f) .
$$

\section{Proof of the theorem and corollaries}

Proof of Theorem 1.1. Since normality is a local property, without loss of generality we may assume that $\mathfrak{D}=\Delta$. Also since $H_{k}(f-a)=H_{k}(f)$, we may additionally suppose that $a=0$. First we suppose that $a_{k}=1$. We now consider the following cases.

CASE I. Let $k \geq 2$ and $d=0$. Suppose that $\mathfrak{F}$ is not normal in $\Delta$. Then by Lemma 2.1 for $\alpha=k$ we can find a sequence $\left\{z_{n}\right\}$ of points with $\left|z_{n}\right|<r$ $(0<r<1)$, a sequence of positive numbers $\varrho_{n} \rightarrow 0$ and a sequence $\left\{f_{n}\right\} \subset \mathfrak{F}$ of functions such that

$$
g_{n}(\xi)=\varrho_{n}^{-k} f_{n}\left(z_{n}+\varrho_{n} \xi\right) \rightarrow g(\xi)
$$

spherically and locally uniformly, where $g$ is a nonconstant meromorphic function in $\mathbb{C}$ and $g$ has no zero of multiplicity less than $k$. Also $g^{\#}(\xi) \leq$ $g^{\#}(0)=k(A+1)+1$ and $g$ is of order at most 2 , where $A=\max \{|b|,|c|\}$.

We now verify that (I) $g$ and $g^{(k)}-b$ share the value $0 \mathrm{IM}$, and that (II) $g^{(k)}$ does not assume the value $c$ in $\mathbb{C}$.

Let $g\left(\xi_{0}\right)=0$. Then by Hurwitz's theorem there exists a sequence $\xi_{n} \rightarrow \xi_{0}$ such that $g_{n}\left(\xi_{n}\right)=0$ for all sufficiently large values of $n$. So for all sufficiently large values of $n$ we get $f_{n}\left(z_{n}+\varrho_{n} \xi_{n}\right)=0$, and so for all sufficiently large values of $n, H_{k}\left(f_{n}\left(z_{n}+\varrho_{n} \xi_{n}\right)\right)=b$. Hence

$$
g_{n}^{(k)}\left(\xi_{n}\right)+a_{k-1} \varrho_{n} g_{n}^{(k-1)}\left(\xi_{n}\right)+\cdots+a_{1} \varrho_{n}^{k-1} g_{n}^{(1)}\left(\xi_{n}\right)=b .
$$

Letting $n \rightarrow \infty$ we obtain $g^{(k)}\left(\xi_{0}\right)=b$. 
Next let $g^{(k)}\left(\eta_{0}\right)=b$. First we verify that $g^{(k)}(\xi) \not \equiv b$. If $g^{(k)}(\xi) \equiv b$ then $g$ becomes a polynomial of degree at most $k$. Since $g$ has no zero of multiplicity less than $k$ and $g$ is nonconstant, it follows that $g$ is a polynomial of degree $k$ and so it has a single zero of multiplicity $k$. Hence we can write

$$
g(\xi)=\frac{b\left(\xi-\xi_{1}\right)^{k}}{k !}
$$

By a simple calculation we deduce from (3.1) that $g^{\#}(0) \leq k / 2$ if $\left|\xi_{1}\right| \geq 1$ and $g^{\#}(0) \leq|b|$ if $\left|\xi_{1}\right|<1$. Therefore $g^{\#}(0)<k(|b|+1)+1$, which is a contradiction.

Since $g^{(k)}\left(\eta_{0}\right)=b$ and $g_{n}^{(k)}(\eta)+a_{k-1} \varrho_{n} g_{n}^{(k-1)}(\eta)+\cdots+a_{1} \varrho_{n}^{k-1} g_{n}^{(1)}(\eta)$ converges uniformly to $g^{(k)}(\eta)$ in some neighbourhood of $\eta_{0}$, by Hurwitz's theorem there exists a sequence $\eta_{n} \rightarrow \eta_{0}$ such that for all large values of $n$,

$$
g_{n}^{(k)}\left(\eta_{n}\right)+a_{k-1} \varrho_{n} g_{n}^{(k-1)}\left(\eta_{n}\right)+\cdots+a_{1} \varrho_{n}^{k-1} g_{n}^{(1)}\left(\eta_{n}\right)=b
$$

and so $H_{k}\left(f_{n}\left(z_{n}+\varrho_{n} \eta_{n}\right)\right)=b$. Therefore for all sufficiently large values of $n$ we get $f_{n}\left(z_{n}+\varrho_{n} \eta_{n}\right)=0$ and so $g_{n}\left(\eta_{n}\right)=0$. Letting $n \rightarrow \infty$ we obtain $g\left(\eta_{0}\right)=0$. Therefore (I) is verified.

Let $g^{(k)}\left(\zeta_{0}\right)=c$. Then as above we can show that $g^{(k)}(\zeta) \not \equiv c$. Since $g_{n}^{(k)}(\zeta)+a_{k-1} \varrho_{n} g_{n}^{(k-1)}(\zeta)+\cdots+a_{1} \varrho_{n}^{k-1} g_{n}^{(1)}(\zeta)$ converges uniformly to $g^{(k)}(\zeta)$ in some neighbourhood of $\zeta_{0}$, by Hurwitz's theorem there exists a sequence $\zeta_{n} \rightarrow \zeta_{0}$ such that for all large values of $n$,

$$
g_{n}^{(k)}\left(\zeta_{n}\right)+a_{k-1} \varrho_{n} g_{n}^{(k-1)}\left(\zeta_{n}\right)+\cdots+a_{1} \varrho_{n}^{k-1} g_{n}^{(1)}\left(\zeta_{n}\right)=c
$$

and so $H_{k}\left(f_{n}\left(z_{n}+\varrho_{n} \zeta_{n}\right)\right)=c$. Therefore $\left|f_{n}\left(z_{n}+\varrho_{n} \zeta_{n}\right)\right| \geq \varepsilon$ and so $\left|g_{n}\left(\zeta_{n}\right)\right| \geq$ $\varepsilon / \varrho_{n}^{k}$ for all large values of $n$. This shows that $g\left(\zeta_{0}\right)=\infty$, which is a contradiction. So (II) is verified.

If $b \neq c$, by Lemma 2.2, $g$ becomes a constant, which is impossible. Let $b=c$. Then from (I) and (II) we see that $g$ does not assume the value 0 and $g^{(k)}$ does not assume the value $c \neq 0$. If $g$ is transcendental, by Lemma 2.6 we get $T(r, g)=S(r, g)$, which is a contradiction. If $g$ is rational, by Lemma $2.5, g$ becomes a constant, which is impossible. Therefore the family $\mathfrak{F}$ is normal.

CASE II. Let $k \geq 2$ and $d \neq 0$. Suppose that $\mathfrak{F}_{1}=\{f-d: f \in \mathfrak{F}\}$. If $\mathfrak{F}_{1}$ is not normal in $\Delta$, by Lemma 2.1 for $\alpha=0$ we can find a sequence $\left\{z_{n}\right\}$ of points with $\left|z_{n}\right|<r(0<r<1)$, a sequence of positive numbers $\varrho_{n} \rightarrow 0$ and a sequence $\left\{f_{n}-d\right\} \subset \mathfrak{F}_{1}$ of functions such that

$$
g_{n}(\xi)=f_{n}\left(z_{n}+\varrho_{n} \xi\right)-d \rightarrow g(\xi)
$$

spherically and locally uniformly, where $g$ is a nonconstant meromorphic function in $\mathbb{C}$ and $g$ has no zero of multiplicity less then $k$. Further $g$ is of order at most 2 . 
We now verify that (III) $g^{(k)}$ does not assume the value 0 in $\mathbb{C}$, and that (IV) $g+d$ does not assume the value 0 in $\mathbb{C}$.

Let $g^{(k)}\left(\xi_{0}\right)=0$ for some $\xi_{0} \in \mathbb{C}$. Also we see that $g^{(k)}(\xi) \not \equiv 0$, for otherwise $g$ becomes a polynomial of degree less than $k$, which is impossible because $g$ is nonconstant and does not have any zero of multiplicity less than $k$.

Since in a neighbourhood of $\xi_{0}$,

$$
g_{n}^{(k)}(\xi)+a_{k-1} \varrho_{n} g_{n}^{(k-1)}(\xi)+\cdots+a_{1} \varrho_{n}^{k-1} g_{n}^{(1)}(\xi)-\varrho_{n}^{k} b
$$

converges uniformly to $g^{(k)}(\xi)$, by Hurwitz's theorem there exists a sequence $\xi_{n} \rightarrow \xi_{0}$ such that for all large values of $n$,

$$
g_{n}^{(k)}\left(\xi_{n}\right)+a_{k-1} \varrho_{n} g_{n}^{(k-1)}\left(\xi_{n}\right)+\cdots+a_{1} \varrho_{n}^{k-1} g_{n}^{(1)}\left(\xi_{n}\right)-\varrho_{n}^{k} b=0,
$$

and so for all large values of $n$ we get $H_{k}\left(f_{n}\left(z_{n}+\varrho_{n} \xi_{n}\right)\right)=b$. Therefore for all large values of $n$ we obtain $f_{n}\left(z_{n}+\varrho_{n} \xi_{n}\right)=0$ and so $g_{n}\left(\xi_{n}\right)+d=0$. Letting $n \rightarrow \infty$ we get

$$
g\left(\xi_{0}\right)+d=0
$$

Again since in a neighbourhood of $\xi_{0}$,

$$
g_{n}^{(k)}(\xi)+a_{k-1} \varrho_{n} g_{n}^{(k-1)}(\xi)+\cdots+a_{1} \varrho_{n}^{k-1} g_{n}^{(1)}(\xi)-\varrho_{n}^{k} c
$$

converges uniformly to $g^{(k)}(\xi)$, by Hurwitz's theorem there exists a sequence $\chi_{n} \rightarrow \xi_{0}$ such that

$$
g_{n}^{(k)}\left(\chi_{n}\right)+a_{k-1} \varrho_{n} g_{n}^{(k-1)}\left(\chi_{n}\right)+\cdots+a_{1} \varrho_{n}^{k-1} g_{n}^{(1)}\left(\chi_{n}\right)-\varrho_{n}^{k} c=0
$$

for all large values of $n$. Hence for all large values of $n$ we deduce that $H_{k}\left(f_{n}\left(z_{n}+\varrho_{n} \chi_{n}\right)\right)=c$. So for all large values of $n$,

$$
\left|f_{n}\left(z_{n}+\varrho_{n} \chi_{n}\right)\right| \geq \varepsilon, \quad \text { i.e., } \quad\left|g_{n}\left(\chi_{n}\right)+d\right| \geq \varepsilon .
$$

Letting $n \rightarrow \infty$ we obtain $\left|g\left(\xi_{0}\right)+d\right| \geq \varepsilon$, which contradicts (3.2). Therefore (III) is verified.

Next let $g\left(\beta_{0}\right)+d=0$. Then by Hurwitz's theorem there exists a sequence $\beta_{n} \rightarrow \beta_{0}$ such that for all large values of $n, f_{n}\left(z_{n}+\varrho_{n} \beta_{n}\right)-d=g_{n}\left(\beta_{n}\right)=-d$ and so $f_{n}\left(z_{n}+\varrho_{n} \beta_{n}\right)=0$. Hence for all large values of $n$ we deduce that $H_{k}\left(f_{n}\left(z_{n}+\varrho_{n} \beta_{n}\right)\right)=b$ and so

$$
g_{n}^{(k)}\left(\beta_{n}\right)+a_{k-1} \varrho_{n} g_{n}^{(k-1)}\left(\beta_{n}\right)+\cdots+a_{1} \varrho_{n}^{k-1} g_{n}^{(1)}\left(\beta_{n}\right)=b \varrho_{n}^{k} .
$$

Letting $n \rightarrow \infty$ we get $g^{(k)}\left(\beta_{0}\right)=0$, which contradicts (III). Therefore (IV) is verified.

Now by Lemma 2.9 we see that either $g(\xi)=-d+e^{A z+B}$ or $g(\xi)=$ $-d+1 /(A z+B)^{m}$. Since $d \neq 0$, it follows that $g$ has only simple zeros, which is impossible. Therefore $\mathfrak{F}_{1}$ and so $\mathfrak{F}$ is normal.

CASE III. Let $k=1$. In this case condition (i) of the theorem is immaterial and so the proof does not depend on $d$. If $\mathfrak{F}$ is not normal in $\Delta$, 
proceeding as Case I we can show that there exists a nonconstant meromorphic function $g$ of finite order such that $g$ and $g^{(1)}-b$ share the value 0 IM and $g^{(1)}$ does not assume the value $c$ in $\mathbb{C}$.

If $b \neq c$ then by Lemma 2.3 we get $b=(1+m) c$ for some positive integer $m$, which is impossible. Let $b=c$. Then $g$ does not assume the value 0 and $g^{(1)}$ does not assume the value $c$. If $g$ is rational, by Lemma 2.5, $g$ becomes a constant, which is impossible. If $g$ is transcendental, by Lemma 2.6 we get $T(r, g)=S(r, g)$, which is a contradiction. Therefore the family $\mathfrak{F}$ is normal.

Finally, suppose that $a_{k} \neq 1$. We now put $G_{k}(f)=\left(1 / a_{k}\right) H_{k}(f), b_{1}=$ $b / a_{k}$ and $c_{1}=c / a_{k}$. Then the leading coefficient of $G_{k}(f)$ is 1 and $b_{1} / c_{1}$ $=b / c$. Also the following hold:

(i) $f-d$ has no zero of multiplicity less than $k$,

(ii) $f-a$ and $G_{k}(f)-b_{1}$ share the value $0 \mathrm{IM}$,

(iii) $|f(z)-a| \geq \varepsilon$ whenever $G_{k}(f)=c_{1}$.

Therefore the family $\mathfrak{F}$ is normal in this case as well by the result for $a_{k}=1$. This proves the theorem.

Proof of Corollary 1.1. Since $c \neq \alpha$, we choose an $\varepsilon$ such that $0<\varepsilon<$ $|c-\alpha|$. Then from condition (iii) we see that if $H_{k}(f)=b$ then $|f(z)-\alpha|=$ $|c-\alpha|>\varepsilon$, which is condition (iii) of Theorem 1.1. Hence the corollary follows from Theorem 1.1.

Proof of Corollary 1.2. Interchanging $a$ and $b$ if necessary, we may choose $|a| \leq|b|$. Since $a \neq b$, it follows that $b \neq 0$ and $a / b$ is not a positive integer. We now choose an $\varepsilon$ such that $0<\varepsilon<|b-a|$. So we see that if $H_{k}(f)=b$ then $|f(z)-a|=|b-a|>\varepsilon$. Hence the corollary follows from Theorem 1.1.

4. Application. In this section we prove a value distribution theorem for a differential polynomial which follows from Theorem 1.1.

THEOREM 4.1. Let $f$ be a transcendental meromorphic function and $a_{1}, \ldots, a_{k} \neq 0$ be constants such that $H_{k}\left(f^{p}\right)=H_{k}\left(f^{p} ; a_{1}, \ldots, a_{k}\right)$ is also transcendental, where $p \geq 2$ is an integer. Let a be a finite complex number such that

(i) $f$ has no zero of multiplicity less than $k / p$,

(ii) $f$ and $H_{k}\left(f^{p}\right)-a$ share the value $0 I M$.

Then for every complex number $b \neq 0, \infty$, the function $H_{k}\left(f^{p}\right)-b$ has infinitely many zeros.

Proof. We consider the following cases.

CASE I. Let $f$ be of infinite order. Then by Lemma 2.10 there exists a sequence $z_{n} \rightarrow \infty$ such that $f^{\#}\left(z_{n}\right) \rightarrow \infty$ as $n \rightarrow \infty$. Let $f_{n}(z)=f\left(z_{n}+z\right)$ 
for $n=1,2, \ldots$. Then $f_{n}^{\#}(0)=f^{\#}\left(z_{n}\right) \rightarrow \infty$ as $n \rightarrow \infty$. So by Marty's criterion no subfamily of $\left\{f_{n}\right\}$ is normal in $\Delta$. Suppose that $H_{k}\left(f^{p}\right)-b$ has a finite number of zeros. Since $z_{n} \rightarrow \infty$ as $n \rightarrow \infty$, there exists a positive integer $N$ such that for $n \geq N, H_{k}\left(f_{n}^{p}\right)-b$ has no zero in $\Delta$. So by Theorem 1.1 the family $\left\{f_{n}: n \geq N\right\}$ is normal in $\Delta$, which is a contradiction. Therefore $H_{k}\left(f^{p}\right)-b$ has infinitely many zeros.

CASE II. Let $f$ be of finite order. If $f$ has only finitely many zeros, by Lemma 2.11 we get

$$
T\left(r, f^{p}\right) \leq \bar{N}\left(r, \infty ; f^{p}\right)+\bar{N}\left(r, b ; H_{k}\left(f^{p}\right)\right)+S\left(r, f^{p}\right)
$$

and so

$$
(p-1) T(r, f) \leq \bar{N}\left(r, b ; H_{k}\left(f^{p}\right)\right)+S(r, f),
$$

which shows that $H_{k}\left(f^{p}\right)-b$ has infinitely many zeros.

Let $f$ have infinitely many zeros, say $w_{1}, w_{2}, \ldots$ We put $g(z)=$ $a_{k} h^{(k-1)}(z)+a_{k-1} h^{(k-2)}(z)+\cdots+a_{1} h(z)-b z$, where $h(z)=\{f(z)\}^{p}$. Let $g^{\prime}(z)=H_{k}\left(f^{p}\right)-b$ have only finitely many zeros. So $g$ has only finitely many critical values and so, by Lemma $2.7, g$ has only finitely many asymptotic values. We assume, without loss of generality, that $g(0) \neq \infty$. Then by Lemma 2.8 there exists $R>0$ such that for $n=1,2, \ldots$,

$$
\left|\frac{w_{n} g^{\prime}\left(w_{n}\right)}{g\left(w_{n}\right)}\right| \geq \frac{1}{2 \pi} \log \frac{\left|g\left(w_{n}\right)\right|}{R}=\frac{1}{2 \pi} \log \frac{\left|b w_{n}\right|}{R},
$$

so that

$$
\left|\frac{w_{n} g^{\prime}\left(w_{n}\right)}{g\left(w_{n}\right)}\right| \rightarrow \infty \quad \text { as } n \rightarrow \infty .
$$

On the other hand, for $n=1,2, \ldots$ we get

$$
\left|\frac{w_{n} g^{\prime}\left(w_{n}\right)}{g\left(w_{n}\right)}\right|=\frac{|a-b|}{|b|}
$$

which is a contradiction. Therefore $H_{k}\left(f^{p}\right)-b$ has infinitely many zeros. This proves the theorem.

Acknowledgements. The authors are thankful to the referee for her/his valuable suggestions.

\section{References}

[1] W. Bergweiler, On the zeros of certain homogeneous differential polynomials, Arch. Math. (Basel) 64 (1995), 199-202.

[2] W. Bergweiler and A. Eremenko, On the singularities of the inverse to a meromorphic function of finite order, Rev. Mat. Iberoamericana 11 (1995), 355-373.

[3] H. H. Chen and M. L. Fang, Shared values and normal families of meromorphic functions, J. Math. Anal. Appl. 260 (2001), 124-132. 
[4] J. Clunie and W. K. Hayman, The spherical derivative of integral and meromorphic functions, Comment. Math. Helv. 40 (1966), 117-148.

[5] M. L. Fang and L. Zalcman, Normal families and shared values of meromorphic functions, Ann. Polon. Math. 80 (2003), 133-141.

[6] G. Frank, Eine Vermutung von Hayman über Nullstellen meromorpher Funktionen, Math. Z. 149 (1976), 29-36.

[7] W. K. Hayman, Meromorphic Functions, Clarendon Press, Oxford, 1964.

[8] X. J. Huang, Normality of meromorphic functions with multiple zeros and shared values, J. Math. Anal. Appl. 277 (2003), 190-198.

[9] I. Lahiri and S. Dewan, Differential polynomials and normality, Demonstratio Math. 38 (2005), 579-589.

[10] J. K. Langley, Proof of a conjecture of Hayman concerning $f$ and $f^{\prime \prime}$, J. London Math. Soc. 48 (1993), 500-514.

[11] W. C. Lin and H. X. Yi, Value distribution of meromorphic function concerning shared values, Indian J. Pure Appl. Math. 34 (2003), 535-541.

[12] X. C. Pang and L. Zalcman, Normality and shared values, Ark. Mat. 38 (2000), 171-182.

[13] - - - Normal families and shared values, Bull. London Math. Soc. 32 (2000), $325-331$.

[14] J. L. Schiff, Normal Families, Springer, 1993.

[15] W. Schwick, Sharing values and normality, Arch. Math. (Basel) 59 (1992), 50-54.

[16] Y. Xu, Normality criteria concerning sharing values, Indian J. Pure Appl. Math. 30 (1999), 287-293.

Department of Mathematics

University of Kalyani

West Bengal 741235, India

E-mail: indr9431@dataone.in
Department of Mathematics Bhairab Ganguly College Kolkata 700056, India E-mail: shyamalidewan@rediffmail.com

Received 23.2.2006

and in final form 31.5.2006 